\title{
Myosin II is not required for Drosophila tracheal branch elongation and cell intercalation
}

\author{
Amanda Ochoa-Espinosa ${ }^{1, *, \S, \pi}$, Stefan Harmansa ${ }^{1, \pm, \S}$, Emmanuel Caussinus $^{2}$ and Markus Affolter ${ }^{1, \pi}$
}

\begin{abstract}
The Drosophila tracheal system consists of an interconnected network of monolayered epithelial tubes that ensures oxygen transport in the larval and adult body. During tracheal dorsal branch (DB) development, individual DBs elongate as a cluster of cells, led by tip cells at the front and trailing cells in the rear. Branch elongation is accompanied by extensive cell intercalation and cell lengthening of the trailing stalk cells. Although cell intercalation is governed by Myosin II (Myoll)-dependent forces during tissue elongation in the Drosophila embryo that lead to germ-band extension, it remained unclear whether Myoll plays a similar active role during tracheal branch elongation and intercalation. Here, we have used a nanobodybased approach to selectively knock down Myoll in tracheal cells. Our data show that, despite the depletion of Myoll function, tip cell migration and stalk cell intercalation ( $\mathrm{SCl}$ ) proceed at a normal rate. This confirms a model in which DB elongation and $\mathrm{SCl}$ in the trachea occur as a consequence of tip cell migration, which produces the necessary forces for the branching process.
\end{abstract}

KEY WORDS: Branching morphogenesis, Cell intercalation, Cell migration, Drosophila, Myosin

\section{INTRODUCTION}

During morphogenesis, a coordinated series of complex events, including cell division, cell shape changes and cell rearrangements underlies the formation of functional tissues and organs. Epithelial cell intercalation is a major morphogenetic mechanism that acts in polarized tissue elongation, e.g. during Drosophila germ-band extension (GBE) (Irvine and Wieschaus, 1994), mouse gastrulation (Yen et al., 2009), and C. elegans intestine (Leung et al., 1999) and Xenopus kidney tube development (Lienkamp et al., 2012). During intercalation, controlled cell neighbour exchange results in tissue extension along one axis and concomitant convergence along the orthogonal axis. Intercalation requires contacts between two adjacent cells to shrink (Fig. S1A, type I configuration), resulting in a configuration where four or more cells contact each other (type II configuration; Bertet et al., 2004; Blankenship et al., 2006). Subsequently, the new contact extends (type III configuration)

\footnotetext{
${ }^{1}$ Biozentrum, University of Basel, Klingelbergstr. 50/70, 4056 Basel, Switzerland. ${ }^{2}$ Institute of Molecular Life Sciences (IMLS), University of Zurich, 8057 Zurich, Switzerland.

*Present address: Department of Biomedicine, University Hospital Basel, Hebelstrasse 20, 4031 Basel, Switzerland. ₹Present address: IBDM, UMR 7288, Aix-Marseille Université and CNRS, 13009 Marseille, France.

§These authors contributed equally to this work

ॠAuthors for correspondence (amanda.ochoa@unibas.ch; markus.affolter@unibas.ch)

(D) A.O.-E, 0000-0003-1943-790X; S.H., 0000-0001-6668-7608; M.A., 0000-00025171-0016
}

Received 6 January 2017; Accepted 5 July 2017 leading to a local extension of the tissue (Bardet et al., 2013; Collinet et al., 2015; Zallen and Wieschaus, 2004).

The Drosophila tracheal system presents a paradigm of epithelial remodelling and elongation through cell intercalation in a tubular organ. The primary branches are monolayered epithelial tubes and form in the absence of cell division in two distinct stages. First, tracheal tip cells (TCs) begin to migrate away from the tracheal sac and pull along several tracheal stalk cells into the developing branch, forming a small bud (Samakovlis et al., 1996). In a second phase, the branches elongate and narrow down due to stalk cell intercalation (SCI) and extensive cell lengthening (Ribeiro et al., 2004) (Fig. S1B). SCI of the primary branches follows similar geometrical rules to intercalation in flat epithelia (Lecuit, 2005). Initially, cells in the bud are arranged in a side-to-side configuration and share intercellular junctions with their opposite neighbour but also with cells located distal and proximal along the branch (type I configuration, see Fig. S1B'). Intercalation is initiated by cells reaching around the lumen and forming an autocellular junction (type II configuration), followed by zipping up of the autocellular junction along the proximal-distal axis of the branch (type III configuration). Therefore, the pair of cells initially located side by side, rearranges to an end-to-end configuration, resulting in branch elongation (Fig. S1B') (Neumann and Affolter, 2006; Ribeiro et al., 2004).

Although the steps of cell and junction rearrangements during intercalation have been described in great detail (see Fig. S1), whether intercalation per se is the driving force leading to branch extension remains debatable. Several studies in epithelial tissues suggest that intercalation is the direct consequence of increased cortical contractility resulting from the dynamics and the localization of MyoII, thereby generating the major force controlling tissue elongation (Bardet et al., 2013; Bertet et al., 2004; Rauzi et al., 2008; Simoes et al., 2010). However, external forces acting on tissue boundaries have also been implicated in tissue elongation. For example, extrinsic pulling forces generated by posterior midgut invagination were linked to Drosophila GBE (Butler et al., 2009; Collinet et al., 2015; Kong et al., 2016; Lye et al., 2015), and also the Drosophila wing is shaped by extrinsic tensile forces (Etournay et al., 2015; Ray et al., 2015). Therefore, tissue elongation is a consequence of a combination of local and tissue-scale forces. During tracheal dorsal branch (DB) elongation, laser ablation studies have shown that highly motile tip cells create a tensile stress during migration, resulting in branch elongation and SCI (Caussinus et al., 2008). Furthermore, Spaghetti squash-GFP, a $\mathrm{GFP} / \mathrm{myosin}$ regulatory light chain fusion protein (Sqh/MRLC), does not localize to the adherens junctions during SCI. Therefore, and in contrast to elongating epithelial sheets in the fly embryo (see above), cell intercalation appears not to be the cause but rather the consequence of epithelial branch elongation in the tracheal system.

Nevertheless, it remains possible that, similar to elongating epithelial sheets, a local tensile force at cell boundaries that is 
produced by MyoII activation, plays an additional active role in DB elongation. Given the prominent role of MyoII during epithelial morphogenesis, its presence in the tracheal system throughout development and its clear role in tracheal placode invagination (Nishimura et al., 2007) and tracheal fusion (Kato et al., 2016), we decided to further investigate the function of MyoII during SCI in the tracheal system. To overcome prior limitations due to MyoII maternal contribution and pleiotropic roles in morphogenesis and cytokinesis, we used a nanobody-based approach that can interfere with MyoII activity in a time- and tissue-specific manner (Caussinus et al., 2012; Pasakarnis et al., 2016). Our results show that, in the absence of actomyosin contractibility, tip cell migration and stalk cell intercalation occur normally. Thus, our data provide functional evidence supporting a model proposing that primary branch elongation in the trachea is driven by tip cell migration and passive stalk cell intercalation, and demonstrate that the primary tracheal branching process is a consequence of cell migration and thus is coordinated by tip cell activity.

\section{RESULTS}

\section{deGradFP efficiently knocks down Myoll in a time- and tissue-specific manner during embryogenesis}

In order to interfere with MyoII function directly at the protein level in a time- and tissue-specific manner, we used the deGradFP method. deGradFP allows for the efficient degradation of GFPfusion proteins and can be used to phenocopy loss-of-function mutations (Blattner et al., 2016; Caussinus et al., 2012; Lee et al., 2016; Nagarkar-Jaiswal et al., 2015; Pasakarnis et al., 2016). Here, we used a null mutant for $s q h$ (Jordan and Karess, 1997) rescued by a Sqh-GFP transgene ( $s q h A X 3 ; s q h-S q h-G F P$ ) (Royou et al., 2004). In this genetic background, we expressed deGradFP using the Gal4/ UAS system to target Sqh-GFP for degradation in different tissues and analysed the resulting phenotypes.

In all the experiments shown, the $s q h A X 3 ; s q h-S q h-G F P$ line was used as a maternal counterpart in our crossing schemes, allowing us to easily introduce a Gal4 driver and UAS-deGradFP from the paternal side (see Fig. S2 for a detailed description of the crossing schemes). As $s q h$ is on the X-chromosome, all male progeny from a cross were hemizygous for $s q h A X 3$ and hence the $s q h-S q h-G F P$ transgene on the second chromosome provided the only source of Sqh protein. In contrast, females expressed both non-tagged and GFP-tagged Sqh. In order to distinguish male embryos from female embryos, we used different approaches depending on the experimental condition. In fixed embryos, we used a monoclonal antibody against Sex lethal (Sxl), which recognizes all somatic cell nuclei in females from nuclear cycle 12 (Bopp et al., 1991). For liveimaging analyses, we used a vestigial red fluorescent reporter (5XQE-DsRed) on the X chromosome that has a stripped pattern of expression in the epidermis from stage 11 and continues to be expressed in the embryo and in larval stages in diverse tissues (Zecca and Struhl, 2007). These two methods, in combination with fluorescent reporters and the obvious signs of Sqh-GFP degradation (see below), allowed us to unambiguously discriminate all possible genotypes.

Initially, we validated the efficiency of deGradFP-mediated knockdown of Sqh-GFP in the lateral epidermis, owing to its imaging accessibility and comprehensive characterization. During dorsal closure (DC), epidermal cells elongate in dorsoventral direction to close a gap that exists in the dorsal epidermis. Along the leading edge of the closing epidermis, an actomyosin cable forms (Fig. S3A) (as shown before by Franke et al., 2005; Kiehart et al., 2000; Pasakarnis et al., 2016; Ducuing and Vincent, 2016) and leading cells project filopodia and lamellipodia dorsally (Fig. S3A, right) (Eltsov et al., 2015). To perturb Sqh function in the embryonic epidermis, we used engrailed-Gal4 (en-Gal4) (Tabata et al., 1992) to restrict expression of deGradFP to the posterior compartment of each segment in $s q h A X 3$; sqh-Sqh-GFP embryos.

deGradFP-mediated Sqh-GFP knockdown resulted in an interruption of the actomyosin cable. However, deGradFP mediated knockdown did not result in the total disappearance of Sqh-GFP, instead Shq-GFP remained in what appears to be inclusion bodies (Fig. S3B, arrowheads). This observation was similar to what was previously reported in the epidermis (Pasakarnis et al., 2016) and the wing imaginal discs (Caussinus et al., 2012). Live imaging of the F-actin reporter Lifeact-Ruby revealed that SqhGFP knockdown males formed thicker and longer filipodia at the leading edge than the control embryos (compare Fig. S3A,B, yellow arrows). However, F-actin was not enriched at the bright Sqh-GFP 'inclusion bodies' (Fig. S3B, asterisk; see also Pasakarnis et al., 2016).

To ensure that Sqh function was indeed lost under these conditions, we used an antibody specifically recognizing the phosphorylated and active form of Sqh (P-MRLC) (Ikebe and Hartshorne, 1985; Jordan and Karess, 1997; Karess et al., 1991). In stage 14 male control embryos, P-MRLC showed apical punctate localization and enrichment at the actomyosin cable (Fig. 1A,B). In knockdown embryos, P-MRLC levels were strongly reduced in all deGradFP-expressing cells, even at the leading edge (Fig. 1C,D). Furthermore, it has been suggested previously that a loss of MyoII function results in cortical relaxation (Mason et al., 2013; Royou et al., 2002; Rozbicki et al., 2015). We therefore investigated whether knockdown of Sqh-GFP resulted in aberrant cell morphology. Indeed, staining for the junctional protein E-Cadherin (E-Cad) revealed that Sqh-GFP knockdown resulted in a significant increase in apical cell surface area (Fig. 1E,F and Fig. S3C,D).

By stage 16, Sqh-GFP knockdown cells managed to contact cells in the contralateral stripes. We observed that in the posterior half of the embryo, cells in the deGradFP-expressing stripes moved forward, displacing the non-deGradFP-expressing cells and excluding them completely from the leading edge while sealing aberrantly with other deGradFP-expressing cells and never with non-deGradFP-expressing cells (Fig. S4B). These results are similar to the ones obtained by the striped expression of a dominant-negative version of Rho1, which is a positive upstream regulator of MyoII (Jacinto et al., 2002), and corroborate two more recent studies using either loss-of-function mutants or deGradFP to disrupt the leading edge actomyosin cable (Ducuing and Vincent, 2016; Pasakarnis et al., 2016). Finally, knockdown of Sqh-GFP in stripes resulted in embryonic lethality in male embryos, while female embryos, which carry a wild-type $s q h$ copy, gave rise to viable progeny (Fig. S4C-E).

In summary, these results consistently show that deGradFPmediated knockdown of Sqh-GFP in the rescue background inactivates MyoII and produces phenotypes consistent with a loss of MyoII (i.e. abnormal epidermal packing, disruption of the actomyosin cable and aberrant epidermal leading edge behaviour) without disrupting other actin-based structures such as filopodia. Furthermore, we have tested a number of other means to inactivate MyoII function but did not find another method that resulted in the dorsal open phenotypes observed when knocking down Sqh-GFP in amnioserosa cells using deGradFP (Table S1). Therefore, we conclude that deGradFP represents the best available tool to address the role of MyoII during cell intercalation in tracheal branch formation. 

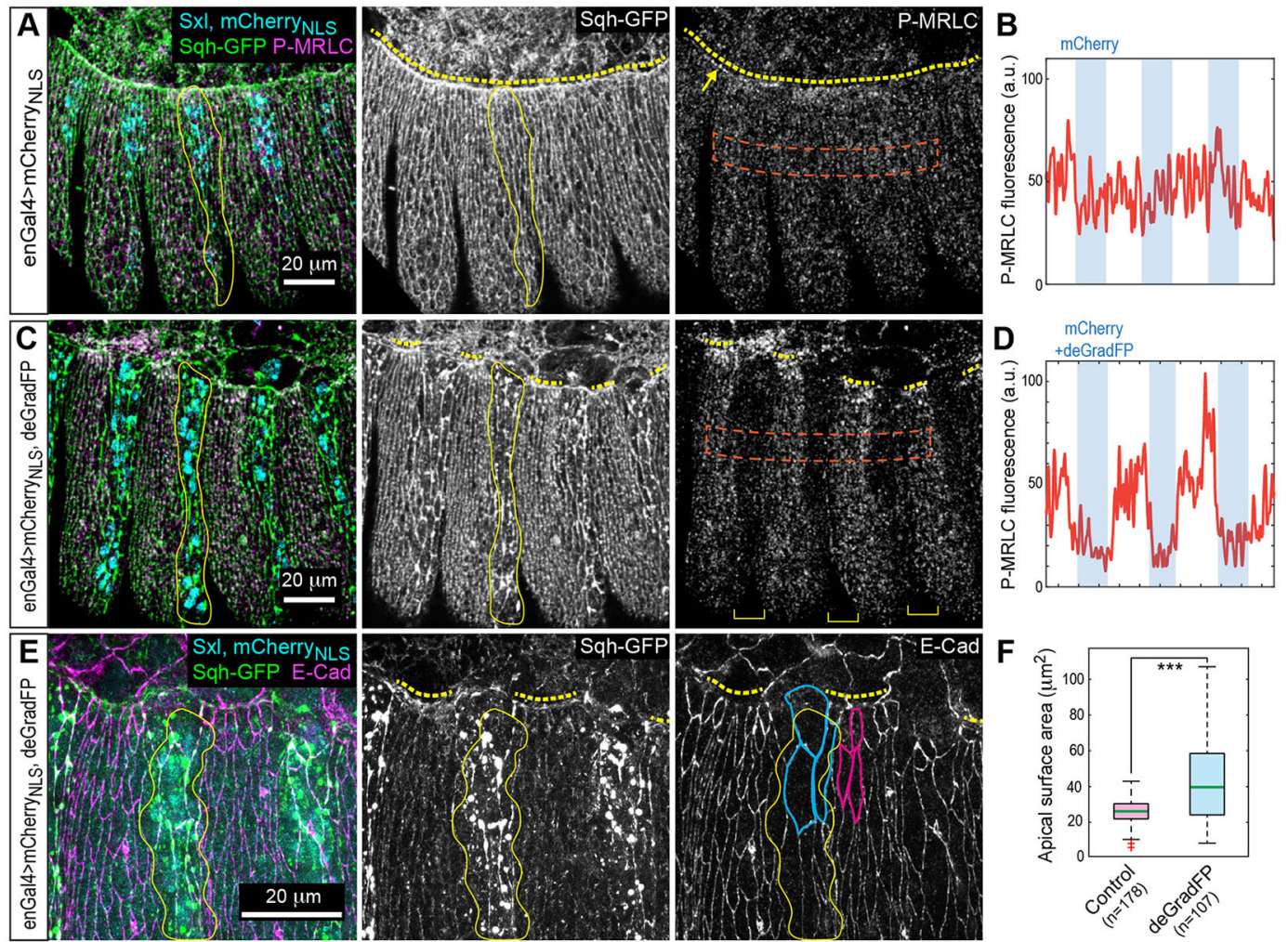

Fig. 1. deGradFP-mediated knockdown of Sqh-GFP in the embryonic epidermis. All images show lateral views of stage 14 male sqhAX3; sqh-Sqh-GFP embryos additionally expressing the indicated transgenes in the engrailed (en::Gal4) stripe pattern. Representative en stripes are highlighted by continuous yellow lines. $(A, B)$ Control embryos stained for phosphorylated MRLC (P-MRLC) show uniform phosphomyosin distribution in all segments and enrichment at the actomyosin cable (arrow in A), although in deGradFP-expressing embryos the P-MRLC signal is drastically reduced in en stripes [see yellow brackets in C (right) and D]. P-MRLC fluorescence levels of the areas marked by a red dotted line in A,C are plotted in B,D, respectively. (E) A deGradFP-expressing embryo stained for E-Cadherin (E-Cad) shows increased apical surface area in the en stripe (blue outlines) compared with cells outside the en stripes (pink outlines).

(F) Quantification of apical cell surface area of cells inside (blue) and outside (pink) the en stripe. The green lines mark the median; whiskers correspond to minimum and maximum data points. Statistical significance was assessed using a two-sided Student's $t$-test ( $\left.{ }^{* * *} P<0.001\right)$; outliers are indicated by a red cross

\section{Tracheal-specific knockdown of Sqh/MRLC does not perturb primary branch elongation}

To determine whether MyoII function is necessary for tracheal system development, we expressed deGradFP under the control of the trachea-specific btl-Gal4 driver in sqh mutant background. btl-Gal4 is expressed in tracheal cells from late stage 11 onwards (Shiga et al., 1996), after the invagination of the tracheal placode (Fig. S5A). Low-magnification time-lapse imaging of knockdown embryos revealed that Sqh-GFP showed a dotted appearance at each tracheomere from early stage 12 , indicating deGradFP activity and efficient inactivation of Sqh-GFP (Movie 1 and Fig. S5B).

In order to verify the efficiency of Sqh-GFP knockdown and to assess the extent of the reduction in MyoII activity in tracheal cells, we performed staining against P-MRLC. In wild-type embryos (Fig. S6) and in female $s q h A X 3 /+$; sqh-Sqh-GFP embryos (carrying one wild-type copy of $s q h$ ) expressing deGradFP, we detected low, rather diffuse levels of P-MRLC along the junctions of tracheal cells (Fig. 2A, white arrows). In contrast, in male sqhAX3; sqh-Sqh-GFP embryos expressing deGradFP, the junctional P-MRLC signal was lost (Fig. 2B). Importantly, we also did not detect a P-MRLC signal in the dotted Sqh-GFP spots (arrowheads in Fig. 2B, bottom). These results show that deGradFP expression provides an effective way to inactivate Sqh-GFP, and hence MyoII function, in tracheal cells.

In the trachea of embryos older than stage 16, we observed that dorsal trunk (DT) morphology was affected by Sqh-GFP knockdown (Fig. S7). Sqh-GFP knockdown embryos showed increased DT diameters and the smooth tube surface observed in control embryos was lost (Fig. S7A,B). Furthermore, knockdown embryos died as first instar larvae (see Fig. S7C). Previous studies highlighted the requirement of MyoII activity for tracheal branch fusions and for extracellular matrix remodelling in tracheal branches (Kato et al., 2016). In line with these findings, the phenotypes we observed in the DT, as well as the larval lethality, are likely to be caused by the lack of MyoII function at developmental stages following DB elongation and SCI. These results further support the efficiency of Sqh-GFP knockdown by deGradFP.

Despite of the lethality and late phenotypes observed in the DT upon loss of MyoII activity in tracheal cells, E-Cad staining in early stage 16 fixed embryos showed that the overall development and the morphology of the tracheal system remained normal upon expression of deGradFP; all major tracheal branches formed, elongated and the majority fused with their corresponding partners (Fig. 2C,D). However, we observed a partially penetrant defect on dorsal branch tube fusion (not quantified), supporting a requirement for MyoII during the fusion process (as shown by Kato et al., 2016). Additionally, the deposition and clearance of Vermiform, a protein essential for normal chitin cable processing (Luschnig et al., 2006) and the subsequent gas filling of the tracheal tubes, proceeded normally in knockdown embryos (Fig. S8 and Movie 2).

To gain a more detailed view of possible consequences of the absence of MyoII activity during cell intercalation, we characterized the dynamics of DB elongation upon Sqh-GFP knockdown in 

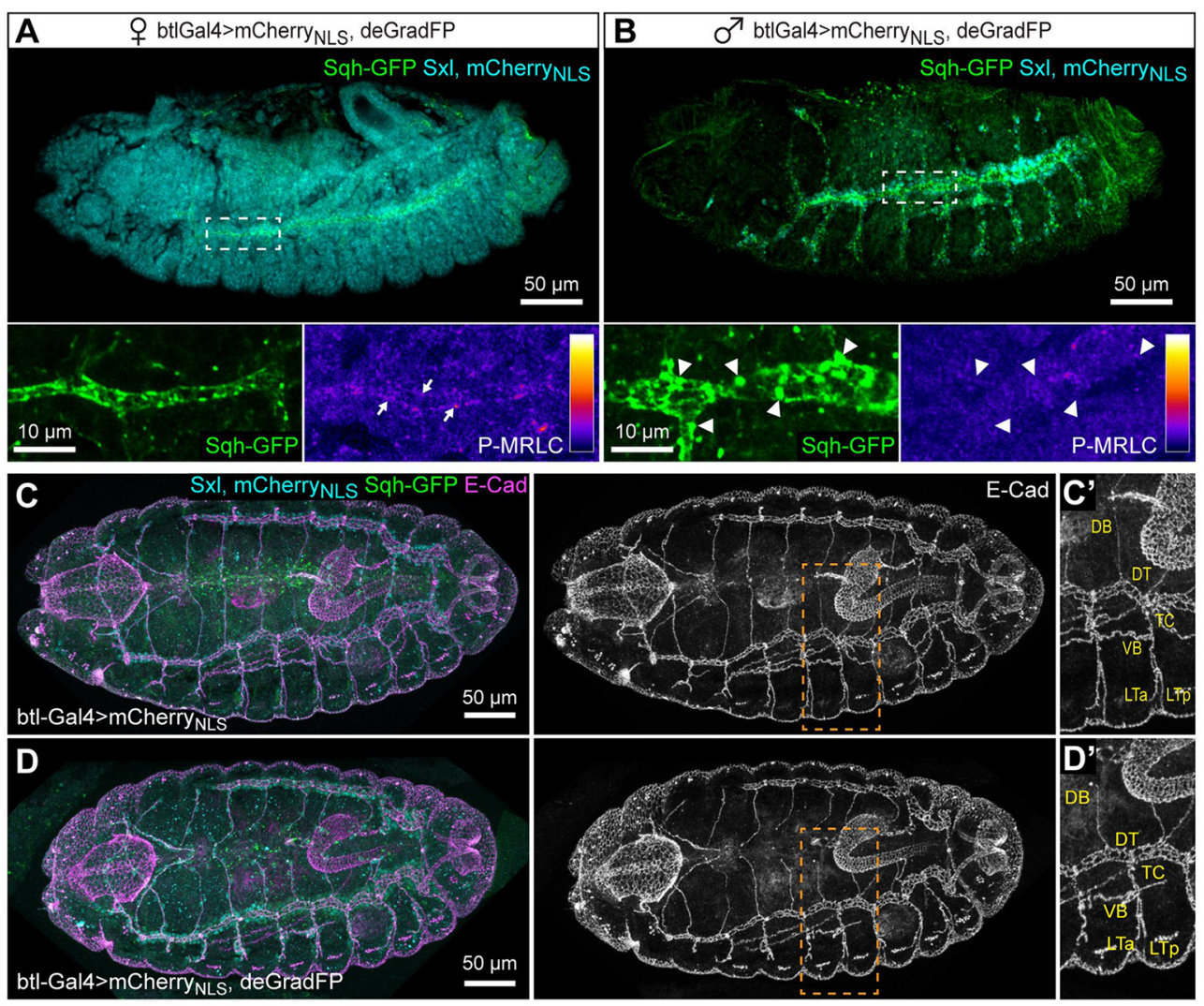

Fig. 2. Sqh-GFP knockdown in the tracheal system results in normal branch architecture. (A,B) Lateral views of stage $14 / 15$ embryos stained for $P$ MRLC and SxI. (A) A female sqhAX3/+; sqh-Sqh-GFP embryo, carrying one wildtype copy of sqh, expressing mCherry NLS and deGradFP in the tracheal system (btlGal4). Low levels of P-MRLC can be visualized along the junctions (arrows). (B) Male sqhAX3; sqh-Sqh-GFP embryo, hemizygous for sqh, expressing mCherry $_{\text {NLS }}$ and deGradFP under btlGal4 control. The P-MRLC signal along the junctions is lost and Sqh-GFP dots also do not show P-MRLC staining (arrowheads). (C,D) Dorsal views of stage 16 male sqhAX3; sqh-Sqh-GFP embryos expressing mCherry NLS $_{\text {(C, control) or }}$ mCherry $_{\text {NLS }}$ together with deGradFP (D) in the tracheal system (btl-Gal4). The trachea system architecture is visualized by staining for E-Cad. In male control (C) and tracheal Sqh knockdown (D) embryos, all main tracheal branches form, elongate and fuse except for few DBs, nevertheless the overall morphology of the tracheal system remains normal. In the higher magnification images $\left(C^{\prime}, D^{\prime}\right)$, the tracheomere 5 ( $\operatorname{Tr} 5)$ branches are labelled: dorsal branch (DB), dorsal trunk (DT), transverse connective (TC), visceral branch (VB) and lateral trunk branches (LTa and LTp). tracheal cells from stage 13/14 onwards (btl-Gal4). Time-lapse movies of male $s q h A X 3$; sqh-Sqh-GFP embryos expressing either mCherry $_{\mathrm{NLS}}$ alone (control) or mCherry NLS $_{\text {and deGradFP }}$ (deGradFP) allowed us to investigate the dynamics of DB elongation (Fig. 3A-D and Movie 3). Under both conditions, five or six DB cells were present in an initial side-by-side configuration (Fig. 3A,B, 0 min; also see Fig. S9). In the following elongation phase, tip cells migrated dorsally (Fig. 3A,B, 35 min) while the stalk cells intercalated, i.e. the cells rearranged to an end-to-end configuration (Fig. 3A,B, $125 \mathrm{~min}$ ). The intercalation process and the dynamics we observed during DB elongation in knockdown embryos were indistinguishable from control embryos (Fig. 3C,D). Furthermore, time-lapse movies of male sqhAX3; sqh-Sqh-GFP embryos expressing either Lifeact-Ruby alone (Control) or LifeactRuby and deGradFP (deGradFP) showed that the tip cells of knockdown DBs formed filopodia (Fig. 3E,F and Movie 4) similar to wild-type embryos (Lebreton and Casanova, 2014; Ribeiro et al., 2004). At later stages, terminal cells formed and the fusion cells contacted the contralateral DB in the Sqh-GFP knockdown embryos, comparable with control embryos (Fig. 3E,F, $160 \mathrm{~min}$ ).

Finally, E-Cad staining on control and knockdown embryos revealed that in stage 16 knockdown embryos (in which nuclei had an end-to-end arrangement), intercellular junctions had remodelled to give rise to autocellular junctions, again as seen in control embryos (Fig. 3G,H). At this final stage, the fusion cells established de novo contacts with the contralateral branches, as visualized by a dot of E-Cad between the two fusion cells under both conditions (Fig. 3G,H). In addition, the spacing between DB nuclei in stage 16 embryos did not significantly differ between the two conditions (Fig. 3I). To verify these observations and ensure that the expression levels of deGradFP were high enough to completely deplete Sqh-GFP by the onset of DB elongation, we also used trachealessGal4 (trh-Gal4), another tracheal driver-line that drives expression from stage 11 on (Fig. S10A), to induce deGradFP expression. Importantly, DB elongation and SCI in Sqh-GFP knockdown embryos using trh-Gal4 was indistinguishable from control embryos (Fig. S10B,C). These results show that MyoII activity is not required during dorsal branch migration and elongation, and, more importantly, that junctional remodelling in SCI proceeds normally in the absence of functional MyoII.

\section{DISCUSSION}

A long-standing question in the field of Drosophila tracheal development was whether MyoII activity is required for DB elongation and SCI. Here, we present data showing that MyoII function is dispensable for branch elongation and concomitant SCI during Drosophila tracheal development. Knockdown of Sqh-GFP, the regulatory light chain of MyoII, specifically in the tracheal system resulted in a normal architecture of the tracheal system and the dynamics of DB elongation and SCI were unaffected. The experiments and results presented here are in line with our previous observations that the pulling forces provided by the tip cells provide enough mechanical force for SCI, and fully support a scenario in which stalk cell intercalation is a cell non-autonomous process brought about by tip cell migration (Caussinus et al., 2008).

Crucial to our approach and different from previous approaches was the use of deGradFP to deplete Sqh-GFP protein in order to block MyoII function. Previous studies mainly relied on hypomorphic mutants and overexpression of dominant-negative and inhibitory proteins in order to interfere with MyoII function. 

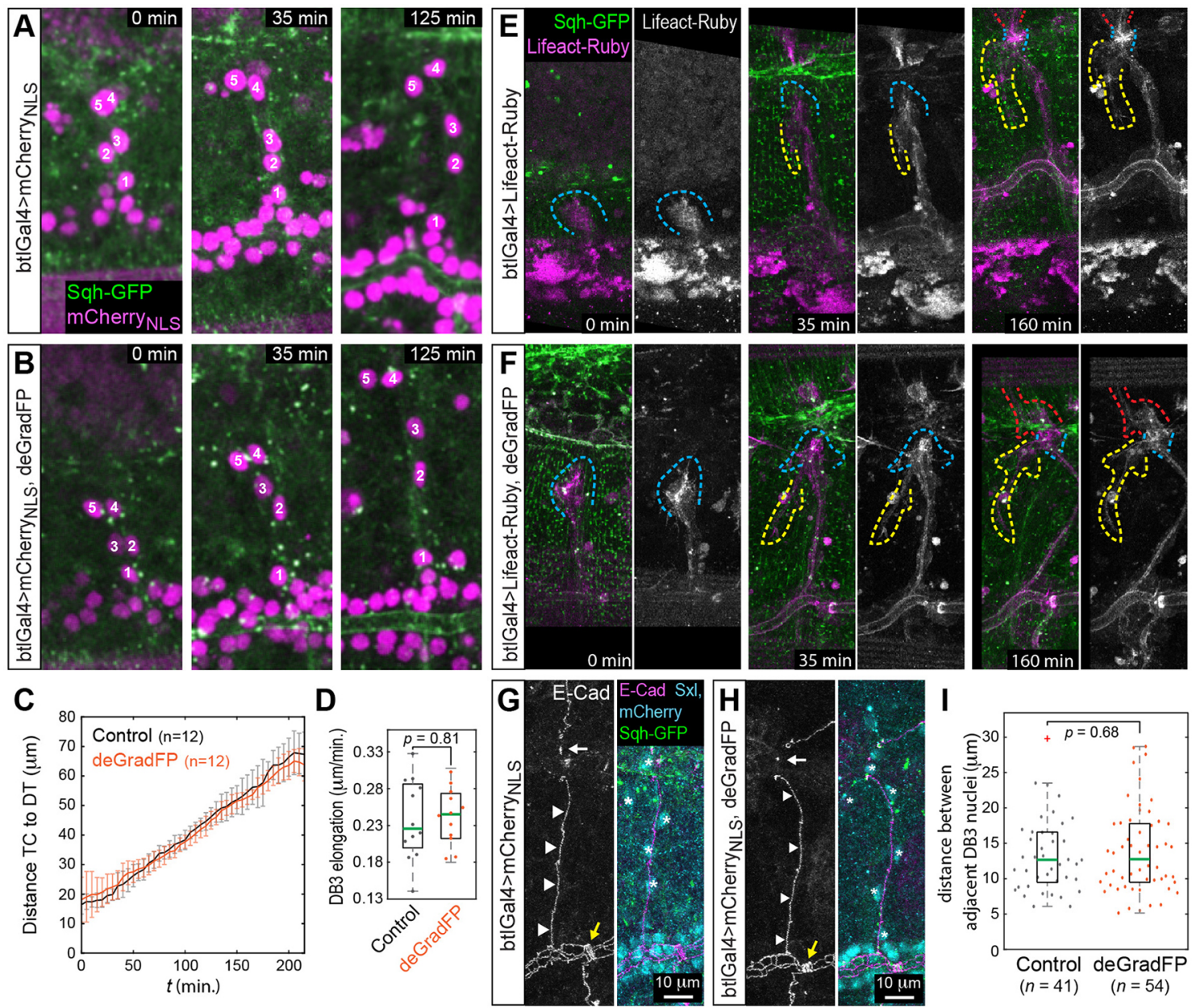

Fig. 3. Dorsal branch elongation and $\mathrm{SCl}$ do not require Myoll activity. Male sqhAX3; sqh-Sqh-GFP embryos expressing the indicated transgenes in the tracheal system (btl-Gal4). (A,B) Frames from time-lapse movies show that in control (A) and Sqh-GFP knockdown (B) embryos, DBs elongate (individual cells are numbered). (C) Distance between the tip cell (TC) and the dorsal trunk (DT) during Tr3 DB elongation. Tr3 DB elongation starts to plateau after 200 min at $\sim 65 \mu \mathrm{m}$ under both conditions. Error bars indicate the standard deviation. (D) Rate of DB elongation. The median is marked by a green line. (E,F) In both control (E) and Sqh knockdown (F) embryos, tip cells extend filopodia (blue dashed lines at 0 min and $35 \mathrm{~min}$ ), form a terminal cell with long cytoplasmic extensions (yellow dashed lines at $35 \mathrm{~min}$ and $160 \mathrm{~min}$ ) and form a fusion cell that eventually contacts the contralateral branch (blue and red dashed lines $160 \mathrm{~min}$ ).

$(G, H)$ Control $(G)$ and Sqh-GFP knockdown $(H)$ stage 16 embryos showing the Tr3 DBs stained for E-Cad. In both cases, cells within the branch have an end-to-end configuration (asterisks over nuclei), form autocellular junctions (arrowheads) and deposit de novo junctional material in contact with the contralateral branch (white arrows). Also highlighted are the fused Tr3 and Tr4 in the DT (yellow arrows). (I) Distance between individual nuclei in the Tr3 DBs of stage 16 embryos. Median (green line), whiskers correspond to minimum and maximum data points. Statistical significance was assessed using a two-sided Student's $t$-test; outliers are indicated by a red cross

However, these approaches have drawbacks that complicate the interpretation of the experimental outcomes. Mutants used to study MyoII function during late embryogenesis must not interfere with maternally contributed mRNA and protein (Franke et al., 2010) in order to allow normal early embryonic development. Hence, owing to protein stability, MyoII function might not be completely lost in such a background. Furthermore, mutations often affect multiple cellular processes and therefore are prone to generate indirect effects or lead to adaptation. To overcome these drawbacks, time- and tissue-specific expression of dominant-negative forms of MyoII or upstream regulators have often been used (Fischer et al., 2014; Franke et al., 2010; Saias et al., 2015). However, these tools seemed to be less efficient in their depleting competence and gave rise to much milder phenotypes than the ones observed with deGradFP (see also Pasakarnis et al., 2016 and Table S1). Therefore, deGradFP is the most effective tool available to deplete Sqh-GFP and interfere with MyoII function in a time- and tissue-specific manner. The results we obtain by deGradFP-mediated inactivation of Sqh-GFP in the tracheal system provide two interesting findings: first, cellular rearrangements during SCI occur normally in the absence of MyoII activity; and second, actomyosin contractile forces are not required in tracheal cells for TC migration and concomitant DB elongation.

The forces that fuel epithelial cell intercalation and tissue elongation have been intensively studied in several organisms. During Drosophila germ-band extension, local forces arising from spatiotemporal dynamics in MyoII levels are required for junctional shrinkage (Bertet et al., 2004; Blankenship et al., 2006; FernandezGonzalez et al., 2009; Levayer and Lecuit, 2013; Rauzi et al., 2008) and subsequent extension (Bardet et al., 2013; Collinet et al., 2015), and act together with global, tissue-scale forces (Butler et al., 2009; Collinet et al., 2015; Etournay et al., 2015; Ray et al., 2015) to drive tissue elongation. Therefore, cell intercalation is a direct consequence of local and tissue-scale forces and is a major cause of tissue elongation in the Drosophila germband. MyoII activity has also been shown to be required for intercalation during chicken primitive streak formation (Rozbicki et al., 2015) and mouse renal 
tube elongation (Lienkamp et al., 2012). Therefore, most intercalation processes mechanistically closely resemble GBE in the Drosophila embryo and use locally produced forces to drive junction and cell-neighbour remodelling. In contrast to the control of intercalation by local force development, external constraints acting on tissue boundaries also control and/or drive intercalation and tissue remodelling. This is very likely the case during Drosophila pupal wing extension, where anchorage of wing blade cells to the pupal cuticle and synchronous contraction of the hinge create a tissue-scale force pattern that drives cellular rearrangements via intercalation and cell division (Etournay et al., 2015; Ray et al., 2015). Our results suggest that despite the resemblance of tracheal SCI to intercalation in the embryonic epidermis (see Fig. S1), the molecular mechanisms underlying force generation in these two systems are fundamentally different.

Pulling forces during tracheal branch elongation, which result in an extrinsic traction force that creates tension in the trailing stalk cells, arise due to TC migration (Caussinus et al., 2008). Cell elongation and rearrangements associated with oriented cell division have been shown to result in stress dissipation (Affolter et al., 2009; Campinho et al., 2013; Guillot and Lecuit, 2013; Wyatt et al., 2015). As tracheal branch elongation occurs in the absence of cell division, extensive cell elongation and cell intercalation presumably provide the only mechanisms for tension relaxation in this context. This is in line with the earlier proposal that cell shape changes and SCI are passive, non-cell-autonomous processes induced by the tension created in stalk cells by TC migration (see also Affolter and Caussinus, 2008; Affolter et al., 2009). Therefore, although during GBE locally produced MyoII-dependent forces drive intercalation and tissue extension, our results show that SCI in the trachea is MyoII independent and that tracheal branch intercalation is, similar to pupal wing extension (Etournay et al., 2015; Ray et al., 2015), a passive process driven by global tissue-scale pulling forces.

Despite the loss of actomyosin activity in all tracheal cells, including the TCs, we found that branch elongation dynamics are unchanged. Therefore, TC migration does not rely on actomyosin contractibility, posing the question: which molecular players might be involved in TC force generation? Interestingly, collective movement of cells depends on actin-based filopodia and lamellipodia (Mayor and Etienne-Manneville, 2016), and was shown in several cases to be independent of MyoII activity (Matsubayashi et al., 2011; SerraPicamal et al., 2012). Furthermore, several studies showed that a downregulation of MyoII is required for effective collective cell migration (Hidalgo-Carcedo et al., 2011; Omelchenko and Hall, 2012; Yamada and Nelson, 2007). It seems that MyoII-independent TC migration might be mainly actin polymerization based and the branching process might therefore be more similar to collective cell migration than to classical epithelial intercalation in flat tissues such as the embryonic epidermis.

Future studies will need to investigate the detailed molecular basis of force generation during trachea TC migration. The extent to which TC migration and DB elongation depend on actin polymerization and the molecules participating in this process might be investigated by directly modulating actin polymerization regulators using deGradFP.

\section{MATERIALS AND METHODS \\ Drosophila stocks}

The following stocks were used: btl-Gal4 (Shiga et al., 1996); UASmCherry $_{\mathrm{NLS}}$ (Caussinus et al., 2008); UAS-LifeAct-Ruby (Hatan et al., 2011); UAS-deGradFP (Caussinus et al., 2012); 5XQEDSRed (Zecca and Struhl, 2007); sqhAX3; sqh-Sqh-GFP (Royou et al., 2004); trh66-Gal4
(Kondo and Hayashi, 2013); a dominant-negative version of zip (UASGFP-DN-zip) (Franke et al., 2005); en-Gal4, amnioserosa-Gal4 ( $\{\mathrm{PGawB}\}$ 332.3), UAS-Dicer2, UAS-shRNA-sqh (TRiP.HMS00437, TRiP. HMS00830 and TRiP.GL00663), UAS-shRNA-zip (TRiP.HMS01618 and TRiP.GL00623) and a dominant-negative version of Rok [UAS-rok. CAT-KG2B1 and UAS-rok.CAT-KG3 (Winter et al., 2001)]; and a dominant-negative version of Rho1 [UAS-Rho1.N19 (Strutt et al., 1997)] (Bloomington Stock Center).

\section{Immunohistochemistry and antibodies}

The following antibodies were used: mouse anti-Sxl-m18 (1:100; DSHB), rabbit anti-phospho-Myosin Light Chain 2 (Ser19) (1:50; 3671, Cell Signaling Technology), rabbit anti-Verm (1:300, a gift from S. Luschnig, WWU Münster) and rat anti-E-Cad DCAD2 (1:100; DSHB). Secondary antibodies were conjugated with Alexa 488, Alexa 568, Alexa 633 (Molecular Probes) or Cy5 (Jackson ImmunoResearch). Embryos were collected overnight and fixed in $4 \%$ formaldehyde in PBS-heptane for $20 \mathrm{~min}$ or $10 \mathrm{~min}$ (for anti-E-Cad) and devitellinized by shaking in methanol-heptane. After extensive washing in methanol and PBT, embryos were blocked in PBT containing 2\% normal goat serum and incubated in primary antibody solution overnight at $4^{\circ} \mathrm{C}$. The next day, embryos were extensively washed with PBT and incubated in secondary antibody solution for $2 \mathrm{~h}$ at room temperature. Subsequently, embryos were washed in PBT again and mounted in Vectashield (H-1000, Vector Laboratories).

\section{Light microscopy}

Imaging was carried out using a Leica TCS SP5 confocal microscope with $\times 20$ dry, $\times 40$ water, $\times 63$ water and $\times 63$ glycerol objectives. For live imaging, embryos were collected overnight, dechorionated in $4 \%$ bleach and mounted in 400-5 mineral oil (Sigma) between a glass coverslip and gaspermeable plastic foil (bioFOLIE 25, In Vitro System and Services) Imaging was carried out at $10 \mathrm{~min}$ intervals for Movies 1 and 2, at $5 \mathrm{~min}$ intervals for Movie 3 and at 2 min intervals for Movie 4. Images were processed using ImageJ (v1.42; NIH) and Imaris (v7.3.0; Bitplane). Timelapse movies were processed using a custom-made plug-in in ImageJ to correct for drift in the $x y$ plane.

\section{Quantifications and statistics}

For the P-MRLC plots in Fig. 1B,D, we measured the fluorescent intensities in the regions of interest indicated in Fig. 1A,C using the Plot Profile function in Image $(\mathrm{NIH})$. Apical cell surface area (quantifications shown in Fig. $1 \mathrm{~F}$ and Fig. S3D) was measured in ImageJ from maximum projections of pre-DC stage 14 embryos stained for E-Cad. We excluded cells from the quantification that we could not clearly assign to either the En-positive (mCherry $_{\mathrm{NLS}}$ ) or the En-negative stripes. To quantify the dynamics of branch elongation (Fig. 3C,D), we measured the direct (minimal) distance between the dorsal trunk and the tip cells of the $\operatorname{Tr} 3 \mathrm{DB}$ in maximum projections of time-lapse movies using ImageJ. The plot in Fig. 3C shows the arithmetic means and the error bars show the standard deviation. For the quantifications in Fig. 3I, live embryos were collected and staged using the completion of dorsal closure as a reference to obtain stage 16 embryos. Live embryos were mounted dorsolaterally as previously described and only embryos in which dorsal branch nuclei appeared in the same plane were imaged at $1 \mu \mathrm{m}$ optical section intervals. $z$ maximum projections of the acquired images were used to measure the distances between nuclei in dorsal branch 3 (which migrates the longest distance in wild type) using ImageJ. $n$ values are indicated either directly in the figures or in the corresponding legend. In the boxplots (Fig. 1F, Fig. 3D,I and Fig. S3D) centre values (green bar) correspond to the median and whiskers mark maximum and minimum data points. A sample number was chosen that was large enough to allow statistical significance to be assessed using a two-sided Student's $t$-test with unequal variance.

\section{Acknowledgements}

We thank the Bloomington Stock Center, the Developmental Studies Hybridoma Bank, Gary Struhl, Stefan Luschnig and Shigeo Hayashi for providing fly stocks and antibodies; the Biozentrum Imaging Core Facility for maintenance of microscopes and support; M. M. Baer, A. Lenard, O. Kanca, F. Hamaratoglu, A. S. Denes and 
M. Müller for helpful discussions and technical assistance; and M. Brauchle for advice and comments on the manuscript.

\section{Competing interests}

The authors declare no competing or financial interests.

\section{Author contributions}

Conceptualization: A.O.-E., E.C., M.A.; Formal analysis: A.O.-E., S.H., E.C.; Investigation: A.O.-E., E.C.; Writing - original draft: A.O.-E., S.H., E.C., M.A.; Writing - review \& editing: A.O.-E., S.H., E.C., M.A.; Visualization: A.O.-E., S.H., E.C.; Funding acquisition: M.A.

\section{Funding}

A.O.-E., E.C. and S.H. were supported by the SystemsX.ch initiative within the framework of the MorphogenetiX project. Work in the lab was supported by grants from cantons Basel-Stadt and Basel-Land, from the Schweizerischer Nationalfonds zur Förderung der Wissenschaftlichen Forschung and from SystemsX.ch (MorphogenetiX to M.A.)

\section{Supplementary information}

Supplementary information available online at

http://dev.biologists.org/lookup/doi/10.1242/dev.148940.supplemental

\section{References}

Affolter, M. and Caussinus, E. (2008). Tracheal branching morphogenesis in Drosophila: new insights into cell behaviour and organ architecture. Developmen 135, 2055-2064.

Affolter, M., Zeller, R. and Caussinus, E. (2009). Tissue remodelling through branching morphogenesis. Nat. Rev. Mol. Cell Biol. 10, 831-842.

Bardet, P.-L., Guirao, B., Paoletti, C., Serman, F., Léopold, V., Bosveld, F., Goya Y., Mirouse, V., Graner, F. and Bellaïche, Y. (2013). PTEN controls junction lengthening and stability during cell rearrangement in epithelial tissue. Dev. Cell 26, 674-674.

Bertet, C., Sulak, L. and Lecuit, T. (2004). Myosin-dependent junction remodelling controls planar cell intercalation and axis elongation. Nature 429,667-671.

Blankenship, J. T., Backovic, S. T., Sanny, J. S. P., Weitz, O. and Zallen, J. A (2006). Multicellular rosette formation links planar cell polarity to tissue morphogenesis. Dev. Cell 11, 459-470.

Blattner, A. C., Chaurasia, S., McKee, B. D. and Lehner, C. F. (2016). Separase is required for homolog and sister disjunction during Drosophila melanogaster male meiosis, but not for biorientation of sister centromeres. PLoS Genet. 12 e1005996.

Bopp, D., Bell, L. R., Cline, T. W. and Schedl, P. (1991). Developmental distribution of female-specific Sex-lethal proteins in Drosophila melanogaster. Genes Dev. $\mathbf{5}$, 403-415.

Butler, L. C., Blanchard, G. B., Kabla, A. J., Lawrence, N. J., Welchman, D. P. Mahadevan, L., Adams, R. J. and Sanson, B. (2009). Cell shape changes indicate a role for extrinsic tensile forces in Drosophila germ-band extension. Nat. Cell Biol. 11, 859-864

Campinho, P., Behrndt, M., Ranft, J., Risler, T., Minc, N. and Heisenberg, C.-P. (2013). Tension-oriented cell divisions limit anisotropic tissue tension in epithelial spreading during zebrafish epiboly. Nat. Cell Biol. 15, 1405-1414.

Caussinus, E., Colombelli, J. and Affolter, M. (2008). Tip-cell migration controls stalk-cell intercalation during Drosophila tracheal tube elongation. Curr. Biol. 18 1727-1734

Caussinus, E., Kanca, O. and Affolter, M. (2012). Fluorescent fusion protein knockout mediated by anti-GFP nanobody. Nat. Struct. Mol. Biol. 19, 117-121.

Collinet, C., Rauzi, M., Lenne, P.-F. and Lecuit, T. (2015). Local and tissue-scale forces drive oriented junction growth during tissue extension. Nat. Cell Biol. 17 1247-1258.

Ducuing, A. and Vincent, S. (2016). The actin cable is dispensable in directing dorsal closure dynamics but neutralizes mechanical stress to prevent scarring in the Drosophila embryo. Nat. Cell Biol. 18, 1149-1160.

Eltsov, M., Dubé, N., Yu, Z., Pasakarnis, L., Haselmann-Weiss, U., Brunner, D. and Frangakis, A. S. (2015). Quantitative analysis of cytoskeletal reorganization during epithelial tissue sealing by large-volume electron tomography. Nat. Cell Biol. 17, 605-614.

Etournay, R., Popović, M., Merkel, M., Nandi, A., Blasse, C., Aigouy, B., Brandl, H., Myers, G., Salbreux, G., Jülicher, F. et al. (2015). Interplay of cell dynamics and epithelial tension during morphogenesis of the Drosophila pupal wing. Elife 4, e07090.

Fernandez-Gonzalez, R., Simoes, S. D. M., Röper, J.-C., Eaton, S. and Zallen, J. A. (2009). Myosin II dynamics are regulated by tension in intercalating cells. Dev. Cell 17, 736-743.

Fischer, S. C., Blanchard, G. B., Duque, J., Adams, R. J., Arias, A. M., Guest, S. D. and Gorfinkiel, N. (2014). Contractile and mechanical properties of epithelia with perturbed actomyosin dynamics. PLoS ONE 9, e95695.
Franke, J. D., Montague, R. A. and Kiehart, D. P. (2005). Nonmuscle myosin II generates forces that transmit tension and drive contraction in multiple tissues during dorsal closure. Curr. Biol. 15, 2208-2221.

Franke, J. D., Montague, R. A. and Kiehart, D. P. (2010). Nonmuscle myosin II is required for cell proliferation, cell sheet adhesion and wing hair morphology during wing morphogenesis. Dev. Biol. 345, 117-132.

Guillot, C. and Lecuit, T. (2013). Mechanics of epithelial tissue homeostasis and morphogenesis. Science 340, 1185-1189.

Hatan, M., Shinder, V., Israeli, D., Schnorrer, F. and Volk, T. (2011). The Drosophila blood brain barrier is maintained by GPCR-dependent dynamic actin structures. J. Cell Biol. 192, 307-319.

Hidalgo-Carcedo, C., Hooper, S., Chaudhry, S. I., Williamson, P., Harrington, K., Leitinger, B. and Sahai, E. (2011). Collective cell migration requires suppression of actomyosin at cell-cell contacts mediated by DDR1 and the cell polarity regulators Par3 and Par6. Nat. Cell Biol. 13, 49-58.

Ikebe, M. and Hartshorne, D. J. (1985). Phosphorylation of smooth muscle myosin at two distinct sites by myosin light chain kinase. J. Biol. Chem. 260, 10027-10031.

Irvine, K. D. and Wieschaus, E. (1994). Cell intercalation during Drosophila germband extension and its regulation by pair-rule segmentation genes. Development 120, 827-841.

Jacinto, A., Wood, W., Woolner, S., Hiley, C., Turner, L., Wilson, C., MartinezArias, A. and Martin, P. (2002). Dynamic analysis of actin cable function during Drosophila dorsal closure. Curr. Biol. 12, 1245-1250.

Jordan, P. and Karess, R. (1997). Myosin light chain-activating phosphorylation sites are required for oogenesis in Drosophila. J. Cell Biol. 139, 1805-1819.

Karess, R. E., Chang, X.-J., Edwards, K. A., Kulkarni, S., Aguilera, I. and Kiehart, D. P. (1991). The regulatory light chain of nonmuscle myosin is encoded by spaghetti-squash, a gene required for cytokinesis in Drosophila. Cell 65 1177-1189.

Kato, K., Dong, B., Wada, H., Tanaka-Matakatsu, M., Yagi, Y. and Hayashi, S. (2016). Microtubule-dependent balanced cell contraction and luminal-matrix modification accelerate epithelial tube fusion. Nat. Commun. 7, 11141.

Kiehart, D. P., Galbraith, C. G., Edwards, K. A., Rickoll, W. L. and Montague, R. A. (2000). Multiple forces contribute to cell sheet morphogenesis for dorsal closure in Drosophila. J. Cell Biol. 149, 471-490.

Kondo, T. and Hayashi, S. (2013). Mitotic cell rounding accelerates epithelial invagination. Nature 494, 125-129.

Kong, D., Wolf, F. and Grosshans, J. (2016). Forces directing germ-band extension in Drosophila embryos. Mech. Dev. 144, 11-22.

Lebreton, G. and Casanova, J. (2014). Specification of leading and trailing cell features during collective migration in the Drosophila trachea. J. Cell Sci. 127 465-474

Lecuit, T. (2005). Adhesion remodeling underlying tissue morphogenesis. Trends Cell Biol. 15, 34-42.

Lee, K.-H., Zhang, P., Kim, H. J., Mitrea, D. M., Sarkar, M., Freibaum, B. D., Cika, J., Coughlin, M., Messing, J., Molliex, A. et al. (2016). C9orf72 dipeptide repeats impair the assembly, dynamics, and function of membrane-less organelles. Cell 167, 774-788.e717.

Leung, B., Hermann, G. J. and Priess, J. R. (1999). Organogenesis of the Caenorhabditis elegans intestine. Dev. Biol. 216, 114-134.

Levayer, R. and Lecuit, T. (2013). Oscillation and polarity of E-cadherin asymmetries control actomyosin flow patterns during morphogenesis. Dev. Cell 26, 162-175

Lienkamp, S. S., Liu, K., Karner, C. M., Carroll, T. J., Ronneberger, O. Wallingford, J. B. and Walz, G. (2012). Vertebrate kidney tubules elongate using a planar cell polarity-dependent, rosette-based mechanism of convergent extension. Nat. Genet. 44, 1382-1387.

Luschnig, S., Bätz, T., Armbruster, K. and Krasnow, M. A. (2006). serpentine and vermiform encode matrix proteins with chitin binding and deacetylation domains that limit tracheal tube length in Drosophila. Curr. Biol. 16, 186-194.

Lye, C. M., Blanchard, G. B., Naylor, H. W., Muresan, L., Huisken, J., Adams R. J. and Sanson, B. (2015). Mechanical coupling between endoderm invagination and axis extension in Drosophila. PLoS Biol. 13, e1002292.

Mason, F. M., Tworoger, M. and Martin, A. C. (2013). Apical domain polarization localizes actin-myosin activity to drive ratchet-like apical constriction. Nat. Cell Biol. 15, 926-936.

Matsubayashi, Y., Razzell, W. and Martin, P. (2011). 'White wave' analysis of epithelial scratch wound healing reveals how cells mobilise back from the leading edge in a myosin-II-dependent fashion. J. Cell Sci. 124, 1017-1021.

Mayor, R. and Etienne-Manneville, S. (2016). The front and rear of collective cell migration. Nat. Rev. Mol. Cell Biol. 17, 97-109.

Nagarkar-Jaiswal, S., Lee, P. T., Campbell, M. E., Chen, K., Anguiano-Zarate, S. Gutierrez, M. C., Busby, T., Lin, W. W., He, Y., Schulze, K. L. et al. (2015). A library of MiMICs allows tagging of genes and reversible, spatial and temporal knockdown of proteins in Drosophila. eLife 4

Neumann, M. and Affolter, M. (2006). Remodelling epithelial tubes through cell rearrangements: from cells to molecules. EMBO Rep. 7, 36-40. 
Nishimura, M., Inoue, Y. and Hayashi, S. (2007). A wave of EGFR signaling determines cell alignment and intercalation in the Drosophila tracheal placode. Development 134, 4273-4282.

Omelchenko, T. and Hall, A. (2012). Myosin-IXA regulates collective epithelial cell migration by targeting RhoGAP activity to cell-cell junctions. Curr. Biol. 22, 278-288.

Pasakarnis, L., Frei, E., Caussinus, E., Affolter, M. and Brunner, D. (2016). Amnioserosa cell constriction but not epidermal actin cable tension autonomously drives dorsal closure. Nat. Cell Biol. 18, 1161-1172.

Rauzi, M., Verant, P., Lecuit, T. and Lenne, P.-F. (2008). Nature and anisotropy of cortical forces orienting Drosophila tissue morphogenesis. Nat. Cell Biol. 10 $1401-1410$

Ray, R. P., Matamoro-Vidal, A., Ribeiro, P. S., Tapon, N., Houle, D., SalazarCiudad, I. and Thompson, B. J. (2015). Patterned anchorage to the apical extracellular matrix defines tissue shape in the developing appendages of Drosophila. Dev. Cell 34, 310-322.

Ribeiro, C., Neumann, M. and Affolter, M. (2004). Genetic control of cell intercalation during tracheal morphogenesis in Drosophila. Curr. Biol. 14, 2197-2207.

Royou, A., Sullivan, W. and Karess, R. (2002). Cortical recruitment of nonmuscle myosin II in early syncytial Drosophila embryos: its role in nuclear axial expansion and its regulation by Cdc2 activity. J. Cell Biol. 158, 127-137.

Royou, A., Field, C., Sisson, J. C., Sullivan, W. and Karess, R. (2004). Reassessing the role and dynamics of nonmuscle myosin II during furrow formation in early Drosophila embryos. Mol. Biol. Cell 15, 838-850.

Rozbicki, E., Chuai, M., Karjalainen, A. I., Song, F., Sang, H. M., Martin, R., Knölker, H.-J., MacDonald, M. P. and Weijer, C. J. (2015). Myosin-II-mediated cell shape changes and cell intercalation contribute to primitive streak formation. Nat. Cell Biol. 17, 397-408.

Saias, L., Swoger, J., D’Angelo, A., Hayes, P., Colombelli, J., Sharpe, J., Salbreux, G. and Solon, J. (2015). Decrease in cell volume generates contractile forces driving dorsal closure. Dev. Cell 33, 611-621.

Samakovlis, C., Hacohen, N., Manning, G., Sutherland, D. C., Guillemin, K. and Krasnow, M. A. (1996). Development of the Drosophila tracheal system occurs by a series of morphologically distinct but genetically coupled branching events. Development 122, 1395-1407.

Serra-Picamal, X., Conte, V., Vincent, R., Anon, E., Tambe, D. T., Bazellieres, E., Butler, J. P., Fredberg, J. J. and Trepat, X. (2012). Mechanical waves during tissue expansion. Nat. Phys. 8, 628-634.

Shiga, Y., Tanaka-Matakatsu, M. and Hayashi, S. (1996). A nuclear GFP/ßgalactosidase fusion protein as a marker for morphogenesis in living Drosophila. Dev. Growth Differ. 38, 99-106.

Simoes, S. D. M., Blankenship, J. T., Weitz, O., Farrell, D. L., Tamada, M., Fernandez-Gonzalez, R. and Zallen, J. A. (2010). Rho-kinase directs Bazooka/ Par-3 planar polarity during Drosophila axis elongation. Dev. Cell 19, 377-388.

Strutt, D. I., Weber, U. and Mlodzik, M. (1997). The role of RhoA in tissue polarity and Frizzled signalling. Nature 387, 292-295

Tabata, T., Eaton, S. and Kornberg, T. B. (1992). The Drosophila hedgehog gene is expressed specifically in posterior compartment cells and is a target of engrailed regulation. Genes Dev. 6, 2635-2645.

Winter, C. G., Wang, B., Ballew, A., Royou, A., Karess, R., Axelrod, J. D. and Luo, L. (2001). Drosophila Rho-associated kinase (Drok) links Frizzled-mediated planar cell polarity signaling to the actin cytoskeleton. Cell 105, 81-91.

Wyatt, T. P. J., Harris, A. R., Lam, M., Cheng, Q., Bellis, J., Dimitracopoulos, A., Kabla, A. J., Charras, G. T. and Baum, B. (2015). Emergence of homeostatic epithelial packing and stress dissipation through divisions oriented along the long cell axis. Proc. Natl. Acad. Sci. USA 112, 5726-5731.

Yamada, S. and Nelson, W. J. (2007). Localized zones of Rho and Rac activities drive initiation and expansion of epithelial cell-cell adhesion. J. Cell Biol. 178, 517-527.

Yen, W. W., Williams, M., Periasamy, A., Conaway, M., Burdsal, C., Keller, R., Lu, X. and Sutherland, A. (2009). PTK7 is essential for polarized cell motility and convergent extension during mouse gastrulation. Development 136, 2039-2048.

Zallen, J. A. and Wieschaus, E. (2004). Patterned gene expression directs bipolar planar polarity in Drosophila. Dev. Cell 6, 343-355.

Zecca, M. and Struhl, G. (2007). Recruitment of cells into the Drosophila wing primordium by a feed-forward circuit of vestigial autoregulation. Development 134 , 3001-3010. 